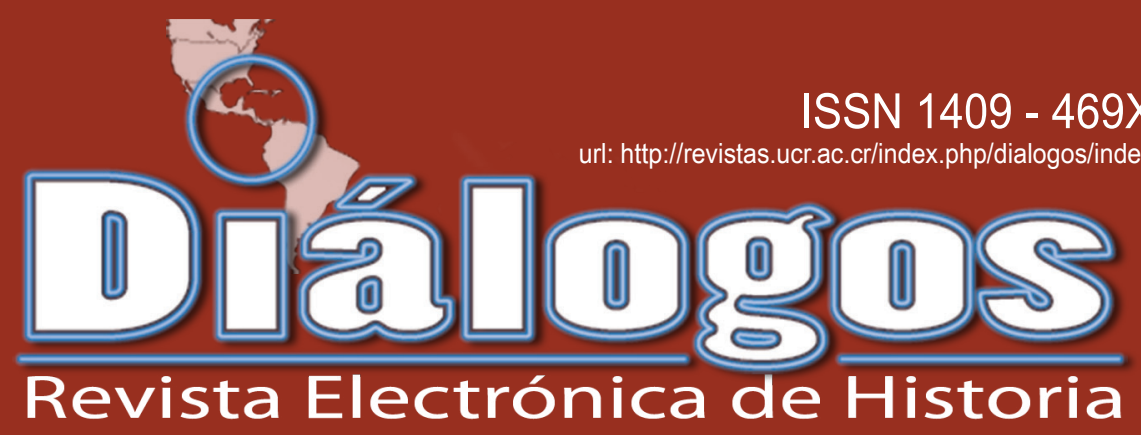

Escuela de Historia. Universidad de Costa Rica Volumen 14 especial en homenaje a Victor Hugo Acuña. Octubre 2013

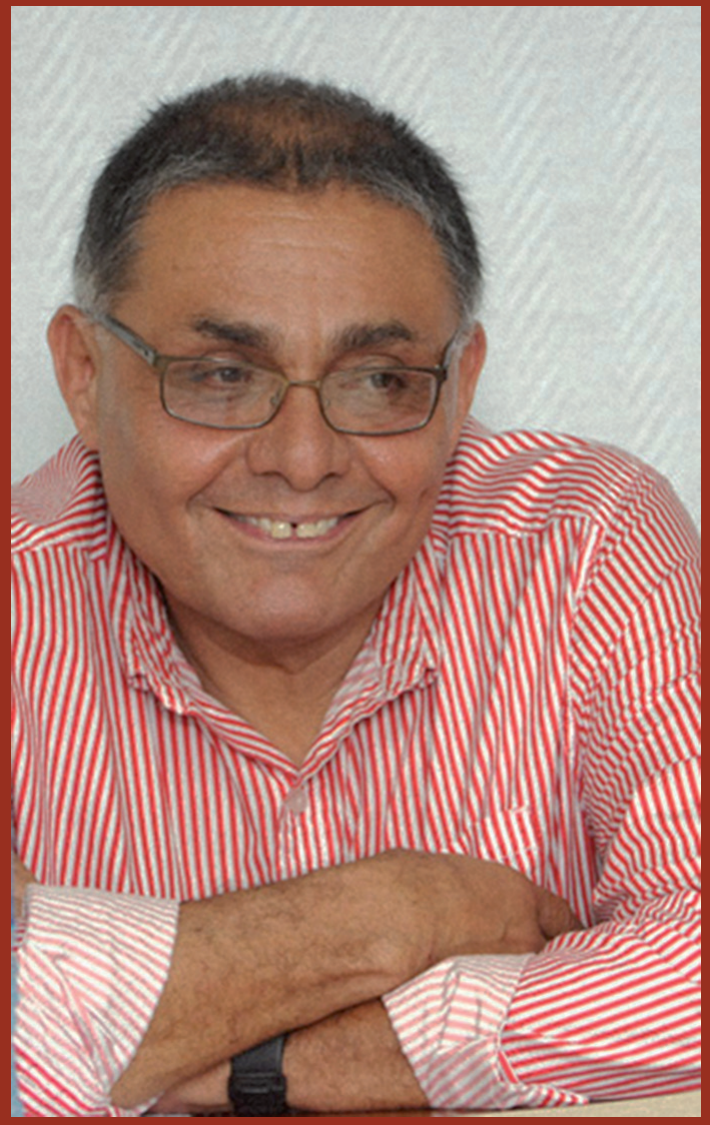

Director de la Revista: Dr. Juan José Marín Hernández juan.marinhernandez@ucr.ac.cr

Editor académico: Dr. Ronny Viales Hurtado - ronny.viales@ucr.ac.cr Editor técnico: M.Sc. Marcela Quirós G. - marcela.quiros@ucr.ac.cr 


\section{Miembros del Consejo Editorial:}

Dr. Juan José Marín Hernández, Catedrático. Director del Centro de Investigaciones Históricas de América Central. Universidad de Costa Rica. Costa Rica. juan. marin@ucr.ac.cr

Dr. Ronny Viales Hurtado. Catedrático. Historia Económica y Social. Universidad de Costa Rica. Director de la Escuela de Historia. Costa Rica. ronny. viales@ucr.ac.cr

Dr. David Díaz Arias: Catedrático. Historia Política, Director del posgrado de Historia y Docente de la Escuela de Historia, Universidad de Costa Rica, Costa Rica.david.diaz@ucr.ac.cr

MSc. Francisco Enríquez. Historia Social. Universidad de Costa Rica. Costa Rica. francisco.enriquez@ucr. ac.cr

Dra. Ana María Botey. Historia de los movimientos sociales. Universidad de Costa Rica. Costa Rica. abotey@gmail.com

\section{Miembros del Consejo Asesor Internacional:}

Dr. José Cal Montoya. Universidad de San Carlos de Guatemala.Guatemala. jecalm@correo.url.edu.gt

Dr. Juan Manuel Palacio. Universidad Nacional de San Martín. Argentina.jpalacio@unsam.edu.ar

Dr. Eduardo Rey. Universidad de Santiago de Compostela. España. ereyt@usc.es

Dr. Heriberto Cairo Carou. Departamento de Ciencia Política y de la Administración III Universidad Complutense de Madrid. España. hcairoca@cps.ucm.es

Dra. Rosa de la Fuente. Departamento de Ciencia Política y de la Administración III Universidad Complutense de Madrid. España. rdelafuente@cps. ucm.es

Dr. Javier Franzé. Departamento de Ciencia Política y de la Administración III Universidad Complutense de Madrid. España. javier.franze@cps.ucm.es

Dr. Jaime Preciado Coronado Departamento de Estudios Ibéricos y Latinoamericanos. Universidad de Guadalajara. México. japreco@hotmail.com
Dr. Gerónimo de Sierra. Vicerrector de la Universidade Federal da Integração Latino-Americana (UNILA) y Departamento de Sociología, Facultad de Ciencias Sociales de la Universidad de la República. Uruguay. geronimo@fcs.edu.uy

Dr. Antonio Palazuelos. Departamento de Ciencia Política y de la Administración III - Universidad Complutense de Madrid. España. palazuelosa@cps. ucm.es

Dr. Werner Mackenbach. Universidad Potsdam. Alemania. werner.mackenbach@uni-potsdam.de

Dr. Guillermo Castro. Ciudad del Saber Panamá. Panamá.gcastro@cdspanama.org

Dra. Natalia Milanesio. University of Houston. Estados Unidos.nmilane2@Central.UH.EDU

Dr. Ricardo González Leandri. Consejo Superior de Investigaciones Científicas - España. España. rgleandri@gmail.com

Dra. Mayra Espina. Centro de Estudios Psicológicos y Sociológicos, La Habana. Cuba.mjdcips@ceniai.inf.cu

Dra. Montserrat Llonch. Departamento de Economía e Historia Económica Universidad Autónoma de Barcelona. España.montserrat.llonch@uab.es

Dra. Estela Grassi. Universidad de Buenos Aires. Argentina. estelagrassi@gmail.com

Dra. Yolanda Blasco. Universidad de Barcelona. España. yolandablasco@ub.edu

Dr. Alfredo Falero. Departamento de Sociología. Universidad de la República. Uruguay. alfredof@adinet. com.uy

\section{Portada:}

Fotografía de Anel Kenjekeeva, Oficina de Divulgación Universidad de Costa Rica, publicada en http://www. ucr.ac.cr/noticias/2012/09/13/escuela-de-historia-rindehomenaje-a-victor-hugo-acuna.html

\section{Equipo Técnico Editorial:}

$\begin{array}{ll}\text { Editora Técnica: } & \text { M.Sc. Marcela Quirós Garita. } \\ & \text { marcela.quiros@ucr.ac.cr } \\ \text { Diagramación: } & \text { Cindy Chaves Uribe } \\ \text { Asistentes: } & \begin{array}{l}\text { Pablo Hurtado Granados } \\ \text { Maureen Méndez Montero }\end{array}\end{array}$


"Diálogos Revista Electrónica de Historia" se publica desde octubre de 1999.

\section{Diálogos está en los siguientes repositorios:}

Dialnet

http://dialnet.unirioja.es/servlet/

revista?tipo_busqueda=CODIGO\&clave_revista $=3325$

Latindex

http://www.latindex.unam.mx/larga.php?opcion=1\&folio=12995;

\section{UCRindex}

http://www.revistas.ucr.ac.cr/

\section{Scielo}

http://www.scielo.cl/

\section{eRevistas}

http://www.erevistas.csic.es/

\section{REDALYC}

http://redalyc.uaemex.mx/src/inicio/FrmBusRevs2.jsp?iEdoRev=2\&cvepai=11;

\section{LANIC}

http://lanic.utexas.edu/la/ca/cr/indexesp.html

Repositorio de Revistas Universidad de Costa Rica http://www.latindex.ucr.ac.cr/

Directorio y recolector de recursos digitales del

Ministerio de Cultura de España

http://roai.mcu.es/es/inicio/inicio.cmd

DOAJ Directory of open access \& Hybrid journals

http://www.doaj.org/doaj?func=byTitle\&hybrid=1\&query=D

Biblioteca de Georgetown

http://library.georgetown.edu/newjour/d/msg02735.html

Asociación para el Fomento de los Estudios Históricos

en Centroamérica

http://afehc. apinc.org/index.php?action=fi_aff\&id=1774

Universidad de Saskatchewan, Canadá

https://library.usask.ca/ejournals/view/1000000000397982

\section{Monografias}

http://www.monografias.com/Links/Historia/more12.shtml

\section{Hispanianova}

http://hispanianova.rediris.es/general/enlaces/hn0708.htm

Universidad del Norte, Colombia

http://www.uninorte.edu.co/publicaciones/memorias/enlaces.htm

Universidad Autónoma de Barcelona

http://seneca.uab.es/historia/hn0708.htm

Repositorio Invenia - Gestión del Conocimiento

http://www.invenia.es/oai:dialnet.unirioja.es:ART0000086144

\section{Enlace Académico}

http://www.enlaceacademico.org/biblioteca/

revistas-en-formato-digital-centroamerica/

\section{Electronic Resources}

http://sunzi1.lib.hku.hk/ER/detail/hkul/3987318

Revistas académicas en texto completo

http://web.prw.net/ vtorres/
Diálogos se anuncia en las siguientes

instituciones y sitios académicos:

Maestroteca

http://www.maestroteca.com/detail/553/dialogos-revista-electronica-de-historia.html

Biblioteca de Georgetown

http://library.georgetown.edu/newjour/d/msg02735.html

Asociación para el Fomento de los Estudios Históricos en Centroamérica

http://afehc. apinc.org/index.php?action=fi_aff\&id=1774

Universidad de Saskatchewan, Canadá

https://library.usask.ca/ejournals/view/1000000000397982

\section{Monografias}

http://www.monografias.com/Links/Historia/more12.shtml

\section{Hispanianova}

http://hispanianova.rediris.es/general/enlaces/hn0708.htm

Universidad del Norte, Colombia

http://www.uninorte.edu.co/publicaciones/memorias/enlaces.htm |

Universidad Autónoma de Barcelona

http://seneca.uab.es/historia/hn0708.htm

Repositorio Invenia - Gestión del Conocimiento

http://www.invenia.es/oai:dialnet.unirioja.es:ART0000086144

\section{Enlace Académico}

http://www.enlaceacademico.org/biblioteca

revistas-en-formato-digital-centroamerica/

\section{Electronic Resources}

http://sunzi1.lib.hku.hk/ER/detail/hkul/3987318

Revistas académicas en texto completo

http://web.prw.net/ vtorres/

La revista electrónica Diálogos es financiada por

Vicerrectoría de Investigación de la Universidad de Costa Rica
Dialnet - eRevistas

- UCRindex - Latindex -

REDALYC - DOJAC - Directorio

y recolector de recursos

digitales del Ministerio de

Cultura de España

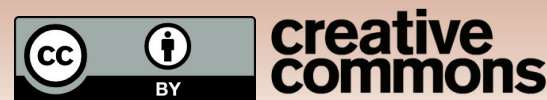





\title{
"UNA FORMA DE HACER HISTORIA DE LA SOCIEDAD EN SU CONJUNTO". CLASES Y SOCIABILIDADES EN LA HISTORIOGRAFÍA DEL DR. VÍCTOR HUGO ACUÑA ORTEGA
}

\section{"A WAY TO DO AN INTEGRAL HISTORY OF SOCIETY". SOCIAL CLASSES AND SOCIABILITY ON VÍCTOR HUGO ACUÑA ORTEGA'S HISTORIOGRAPHY.}

George I. García

\author{
Palabras clave \\ Historiador, Oficio, Clase Social \\ Keywords \\ Historian Craft, Social Classes
}

Fecha de recepción: 17 de mayo, 2013 - Fecha de aceptación: 8 de agosto, 2013

\section{Resumen}

Este artículo se refiere a la producción de Víctor Hugo Acuña en dos sentidos: sobre sus concepciones e influencias teórico-metodológicas, y sobre sus conclusiones sustantivas. Finalmente, esboza algunas consideraciones críticas a modo de balance.

\begin{abstract}
This essay analyzes Víctor Hugo Acuña's studies from two points of view: from his methodological and theoretical influences and from his substantial thesis. It finally draws conclusions based on some critical remarks.
\end{abstract}





\section{"UNA FORMA DE HACER HISTORIA DE LA SOCIEDAD EN SU CONJUNTO". \\ CLASES Y SOCIABILIDADES EN LA HISTORIOGRAFÍA DEL DR. VÍCTOR HUGO ACUÑA ORTEGA}

George I. García

Entre las memorias académicas que conservo con cierta claridad sobre mis primeros años universitarios, figura el curso de Teoría de la historia social y de las mentalidades colectivas, en el segundo semestre del ya lejano 1993. En aquella ocasión, el curso abordó la historia y problemáticas de tales campos de investigación, abonando decisivamente a mi comprensión sobre teoría y metodología de la pesquisa historiográfica, y generándome nuevas inquietudes sobre esas perspectivas. En retrospectiva, no me cabe duda de que mi interés teórico e historiográfico por el estudio de lo cotidiano ha sido parcialmente deudatario de esa historia desde abajo sobre la cual versó buena parte de aquel curso. Con ese curso, además, obtuve las bases para el análisis de textos historiográficos, uno de los fundamentos críticos de la labor del historiador en esta época profusa en cuanto a discursos sobre el pasado (Noiriel, 1997, pp. 15-24).

Dado el contexto de este escrito, ya supondrán el lector -y con toda razónque dicho curso era impartido por el Doctor Víctor Hugo Acuña Ortega. No quisiera traer esta anécdota a colación, empero, como una mera evocación nostálgica, sino más bien como antecedente del tema sobre al cual he escogido extenderme aquí: clases y sociabilidades en la historiografía del Dr. Acuña. Esta exposición sobre estos aspectos -que estimo centrales en la obra del editor del cuarto tomo de la Historia general de Centroamérica- tiene sus raíces más profundas, pues, en mis primeras aproximaciones a su trabajo docente e investigativo.

En este sentido, me referiré a la producción de este nuestro homenajeado investigador en dos sentidos: sobre sus concepciones e influencias teórico-metodológicas, y sobre sus conclusiones sustantivas. Finalmente, esbozaré algunas consideraciones críticas a modo de balance. No huelga indicar que, con la excepción de su artículo sobre la Sociedad La Concordia, los artículos del Dr. Acuña a los que me refiero a continuación son anteriores al segundo lustro de la década de 1990; en esa medida, no pretendo atribuirle posiciones con las que pudiera eventualmente no concordar hoy en día. 


\section{ENFOQUES E INTERLOCUTORES TEÓRICO-METODOLÓGICOS}

Me refiero primeramente, pues, a los autores con los que el Dr. Acuña ha dialogado en el ámbito teórico y metodológico. Tales interlocutores son variados, y se han ido diversificando con los años, de modo que indicaré aquí sólo los que considero más significativos en cuanto a los temas de la presente exposición. Estos interlocutores se han movido, en lo fundamental, dentro de aquella "perspectiva que considera el conflicto social como consustancial a la evolución histórica" (Acuña, 1993, p. 256), un circunloquio formulado en el contexto del aparentemente triunfante neoliberalismo de inicios de los noventas, pero que recuerda a los utilizados por Gramsci al escribir durante el fascismo. Para aquella época indicaba que al estudio de las clases subordinadas le era inherente un valor ético y epistemológico (Acuña, 1996, p. 148), aunque algunos años después, con tono pesimista escribía que "ya no es evidente que la historia tenga una misión política específica e inmediata" (Acuña, 2007, p. 36).

El análisis de clases y sociabilidades ha remitido en la historiografía de Acuña a autores que asimilaron el marxismo, en su recepción británica o en versiones sincretizadas con la École des Annales. En cuanto a los británicos, los estudios de Hobsbawm, Rudé y Thompson sobre clases y movimientos sociales son referencias habituales en el tratamiento del Dr. Acuña, y forman parte esencial de sus análisis de los nacionalismos en Centroamérica; en cuanto al marxismo de la Escuela Francesa, como veremos más adelante, destaca Maurice Agulhon.

En particular, nuestro investigador ha adaptado la conceptualización de Thompson sobre el desarrollo de las clases sociales en el capitalismo. No desearía yo dejar la impresión de que esta influencia se reduce a una fórmula que, como indicaba el propio Acuña, fue repetida casi ad nauseam en la historia social de los años ochentas; con todo, para efectos de síntesis, valga recordar las palabras del autor de Whigs and hunters: "por clase entiendo un fenómeno histórico que unifica una serie de sucesos dispares y aparentemente desconectados, tanto por lo que se refiere a la materia prima de la experiencia, como a la conciencia. Y subrayo que se trata de un fenómeno histórico. [...] Y la clase cobra existencia cuando algunos hombres, de resultas de sus experiencias comunes (heredadas o compartidas), sienten y articulan la identidad de sus intereses a la vez comunes a ellos mismos y frente a otros hombres cuyos intereses son distintos (y habitualmente opuestos) a los suyos" (Thompson, 1989, pp. xiii-vix).

En la obra de Acuña las clases sociales cobran identidad, precisamente, en los conflictos. Así mostró, por ejemplo, cómo los pequeños y medianos productores de café, cuya historia se había convertido en mito de una edad de oro ajena a las contradicciones de clase, debieron movilizarse una vez tras otra, en épocas de crisis tanto como en las de bonanza, contra una burguesía que acaparaba el 
crédito, el procesamiento y la comercialización del grano de oro hacia el extranjero. Del mismo modo, sus aproximaciones a las clases populares de las ciudades costarricenses -la "plebe urbana"- durante las Huelgas de 1920, y al proletariado rural en Limón durante la huelga de 1934, indican cómo es merced a las luchas que se consolidan las identidades de clase. Incluso en su más reciente artículo sobre la Sociedad La Concordia, compuesta por personas de clase media salvadoreña, muestra cómo esa conflictividad era sublimada mediante rituales, himnos y otros símbolos mediante los cuales sus miembros se distanciaban de las clases más subalternas, y manifestaban su deferencia hacia los sectores dominantes (Acuña, 2004, pp. 47-48).

De esta conceptualización afín a Thompson se sigue también la centralidad de la construcción cotidiana del tejido social; así, las sociabilidades son un factor directamente vinculado con la existencia de las clases sociales, y en esa medida constituyen una aproximación indispensable para el estudio de la dinámica social. La historia social no puede entonces prescindir de la consideración sobre los actores sociales concretos, pues son ellos quienes (re)producen las estructuras sociales no tangibles. Omitir las prácticas de los actores sociales lleva, tarde o temprano, a hipostasiar las estructuras, como si existieran más allá de los sujetos, independientemente de ellos. Pero, a la inversa, omitir las estructuras que condicionan a esas prácticas llevan a un voluntarismo abstracto o a un fetichismo del azar y de lo indecidible (Grüner, 2002); a la disolución de la posibilidad de la historia como ciencia social. La célebre frase de Marx de que los seres humanos hacen su historia, pero en condiciones involuntarias, ya dadas (Marx, 2003, p. 33), no puede reducirse a una de sus partes sin perder su sentido.

El concepto de sociabilidad cumple esa función mediadora entre lo cotidiano y las estructuras sociales; es necesario para el análisis de la clase como formación, esto es, como proceso. Este concepto es adoptado por Acuña a partir de los estudios de Maurice Agulhon, según quien la sociabilidad sería "la aptitud de vivir en grupos y consolidar los grupos mediante la constitución de asociaciones voluntarias" (Agulhon, 1994, p. 55). Los estudios de Agulhon sobre las sociabilidades de la clase obrera y las de la burguesía le prestan especial atención a los espacios en los que se ejercían tales prácticas: "no existe asociación, ya sea informal (simple reunión de parroquianos) o formal (con estatutos, reglas escritas), sin que exista un lugar de reunión estable" (Agulhon, 1994, p. 56 y Agulhon, 2009, pp. 30-43).

En los abordajes de Acuña el espacio es, en efecto, un factor determinante en la formación de la clase. Así sucede con el club social La Concordia, anteriormente mencionado, pero también con los espacios laborales: "el mundo del taller era un espacio en donde se forjaba una identidad, una cultura y una conciencia obrera. La vida cotidiana en el taller era una condición y expresión de esa cultura. En el lugar de trabajo se combinaba la palabra con el juego, la burla con la fraternidad; 
se afirmaba la libertad y la autonomía del obrero; se tomaba conciencia de la explotación, se resistía al patrón y también se le consentía. En la cotidianidad del taller se forjaban identidades, resistencias y utopías. El zapatero aprendía a ser distinto y a considerarse diferente" (Acuña, 1991, p. 191).

La investigación sobre las sociabilidades de clase es igualmente emprendida mediante la revisión de la fundación de asociaciones -ya sea para reivindicar derechos o para el esparcimiento-, cuyo análisis temporal, espacial y programático le permite al Dr. Acuña lanzar hipótesis sobre la cohesión y la conciencia de clase. Esto podemos observarlo en su tratamiento de las organizaciones de artesanos y obreros en Costa Rica (Acuña, 1984 y Acuña, 1986) y en Centroamérica (Acuña, 1993, pp. 274-286), así como respecto a las asociaciones de los pequeños y medianos productores de café (Acuña, 1985 y Acuña, 1986). Acuña muestra cómo los cambios y continuidades en el carácter de estas organizaciones, sus objetivos, acciones y declaraciones, manifiestan a la vez transformaciones en la concepción que de sí mismas han tenido las clases populares. Mutuales, sindicatos, confederaciones nacionales e internacionales son distintas formas de asociación que remiten a las dinámicas internas tanto como a las mundiales, a lo largo de las diversas coyunturas.

La clase es el criterio de entrada también para las pesquisas de este autor sobre las identidades nacionales a mediados de los noventas; mediante el análisis de clase determina diversos mecanismos de producción del nacionalismo. El estudio de dichas identidades tiene como trasfondo la teoría del nacionalismo formulada por Benedict Anderson, pero filtrada por las consideraciones de Hobsbawm y de Kiernan sobre la funciones que en él cumplen las clases subalternas; en palabras de Acuña, "en los procesos de formación de las clases trabajadoras, la lealtad nacional y la solidaridad de clase se desarrollan en forma paralela y anudada. No sólo coexisten, sino que es posible que la una no pueda formarse sin la otra, pero, invariablemente, la conciencia de clase es subsidiaria frente a la conciencia nacional. Esta última adopta diversas expresiones en un abanico que va desde las formas más altruistas de internacionalismo, anti-imperialismo y civismo nacional hasta las desviaciones más sórdidas de chauvinismo, xenofobia y racismo" (Acuña, 1994, p. 149).

No está de más indicar que la labor historiográfica del Dr. Acuña ha eludido las simplificaciones y esquematismos propios de una mala aplicación de la teoría; ha partido de que la teoría es un criterio heurístico por ser esclarecido mediante el trabajo empírico del historiador, pero no palabra final (Acuña, 1991, p. 125 y Acuña, 2007, p. 31). En cuanto a las fuentes por él utilizadas, es menester indicar su variedad. Además de las secundarias -naturalmente, de mayor peso en sus textos sobre el conjunto de Centroamérica-, ha recurrido a la prensa comercial, periódicos oficiales y otros documentos formales e informales de los respectivos contextos espacio-temporales en estudio. Del mismo modo, pudo recrear diversos aspectos 
de las condiciones de vida cotidiana de los trabajadores del calzado gracias a la historia oral y a testimonios literarios.

Creo importante señalar que los estudios del autor de Historia e incertidumbre sobre sociabilidades de clase no excluyen sino que suponen y complementan a aquellos anteriores suyos, más centrados en los aspectos de estructuración económica. A éstos no me refiero ahora, empero, pues corresponden al tema de la exposición del Dr. Viales.

\section{APORTES EMPÍRICOS}

En cuanto a las tesis sustantivas que sobre clases y sociabilidades ha aportado la historiografía del Dr. Acuña a la historia social costarricense y centroamericana, las divido en dos series, según correspondan a contextos nacionales o a la región centroamericana en su generalidad. En primer lugar, resalta la diversidad de formas de organización entre las clases subalternas que ha estudiado nuestro homenajeado, y que se desprenden de las diferencias entre sus condiciones de vida: ha mostrado la complejidad de las clases subalternas, y de sus acciones políticas. La cercanía de los salvadoreños clasemedieros con la alta burguesía de su país dista de la beligerancia -en el fondo conservadora - de los pequeños y medianos productores ticos de café, pero también de los radicalizados obreros del banano en el Caribe. En sus estudios sobre las grandes huelgas de 1920 y 1934 en Costa Rica, explora cómo se articularon sectores plurales para converger en luchas específicas; en estos textos lo popular se dice de muchos modos.

También ha demostrado cómo las luchas sociales marcan las coordenadas de la acción estatal. En estos estudios, la política, sea reivindicativa o de otro tipo, tiene sus bases en las relaciones cotidianas, cara a cara, las cuales generan los lazos sociales que se canalizan a través de diversos mecanismos - por ejemplo, negociación, lucha y clientelismo- utilizados por los actores sociales para mantener y mejorar sus condiciones de vida. A partir de las luchas populares, por ejemplo, surgen las grandes reformas estatales; de allí que "la democracia costarricense ha sido forjada en el yunque de la contradicción social, de la contraposición de clases sociales que se reconocen como distintas. [...] La democracia costarricense no es más que una original solución, en el contexto centroamericano, producida por nuestra lucha de clases" (Acuña, 1991, p. 176). Para el caso de Costa Rica, particularmente - y aunque hay que matizar la afirmación de Acuña de que el intervencionismo estatal surge en los treintas (Palmer, 1999, Palmer, 2002, Molina, 2005, pp. 193-206 y Viales, 2005, pp. 71-100) - , indicaba la importancia de la década de los treintas para explicar "los ocho años" de conflictos en la década siguiente 
(Acuña, 1991, p. 175), una labor que ha sido abordada más bien recientemente (Molina, 2007 y Arias, 2011).

Sus ensayos sobre clases subalternas en Centroamérica, por otra parte, tuvieron para entonces un carácter exploratorio. Entre las conclusiones de estos estudios resalta el carácter fuertemente nacionalista de las clases obreras en el istmo; para la época de los liberales, las agrupaciones obreras se identificaban concéntricamente: primero con su país, luego con Centroamérica, finalmente con América Latina (Acuña, 1994, p. 159). En esa medida, los proyectos nacionalistas "liberales" tuvieron un éxito matizado, según las circunstancias locales, por su capacidad de integración de la población rural.

En esa población rural hallaba el Dr. Acuña, precisamente, la capacidad de romper con los proyectos hegemónicos en la región, a través de las organizaciones campesinas en Costa Rica, las milicias de Sandino, mientras que "en El Salvador y Guatemala, cuando las organizaciones obreras influidas por los comunistas y los anarquistas intentaron acercarse a la población campesina para organizarla y movilizarla, sin duda pusieron en peligro intereses fundamentales" (Acuña, 1993, p. 317).

El fundamento económico de las formaciones sociales centroamericanas era bien sabido por las élites de aquellos tiempos: "el régimen liberal pudo tolerar una cierta articulación de los intereses del mundo laboral urbano, pero no soportó en lo más mínimo un proceso similar en el campo. Ese era su límite y esa fue la seña de identidad de un tipo de economía de mercado y de una cierta forma de funcionamiento del mercado mundial que no pudieron existir de una manera menos inhumana" (Acuña, 1993, p. 318).

En los casos de los estudios sobre clases en Costa Rica y El Salvador, así como en los de Centroamérica en conjunto, Acuña ha vuelto la mirada hacia las prácticas sociales de los actores concretos. En esa medida, ha podido mostrar los mecanismos específicos que siguieron, el por qué de sus éxitos y fracasos, así como los alcances de sus acciones. Ha logrado detallar cómo la historia de las sociedades centroamericanas ha sido conformada mediante las solidaridades y los conflictos de clases, desde los cuales ha logrado, ciertamente, "una forma de hacer historia de la sociedad en su conjunto" (Acuña, 1991, p. 197).

\section{EPÍLOGO}

Los estudios de Víctor Hugo Acuña ya forman parte del acerbo indispensable de la historiografía de nuestra región. Son punto de partida necesario, bases actuales para la "erudición de la historia social" de la que hablaba el autor a mediados de los noventas. Hoy, la historia social en Costa Rica está consolidada, lo 
cual se manifiesta en que la "división celular de la historia social" ha ido llenando los vacíos que en aquel momento indicaba que era pertinente colmar: la especialización de la historia de las mujeres, la historia regional, el desarrollo de una nueva historia cultural y una mirada que incorpora a los procesos del resto de Centroamérica en su consideración (Acuña, 1996, pp. 148-149).

En aquel momento apuntaba una falencia de la historia social costarricense que perdura hasta nuestros días: el vacío en el estudio sobre las élites (Acuña, 1996, p. 149). En este sentido, llama la atención que Acuña ha estudiado las clases medias urbanas y rurales, así como a obreros, artesanos y proletarios, pero en sus análisis de clases hasta ahora ha faltado como objeto la burguesía. No sé si será de su interés retomar la línea de investigación sobre clases sociales, pero creo que la pesquisa sobre las élites, siempre presentes en sus investigaciones sobre clases, aunque más bien a la manera de sombras en el fondo del escenario, ayudaría a aclarar varios aspectos de la historia social de Centroamérica. Al fin y al cabo, la del homenajeado sigue siendo una obra abierta, no sólo en el sentido de Umberto Eco, sino en cuanto a que confío en que le quedan todavía mucha páginas por llenar con los frutos de futuras investigaciones.

Finalmente, quisiera plantear la necesidad de retomar el estudio de las relaciones entre estructuración socio-económica, tal como aparecen en "El desarrollo del capitalismo en Costa Rica", y las condiciones de sociabilidad a las cuales nos hemos referido con más detenimiento en esta intervención. El concepto de clase de Thompson es muy útil en este nivel más inmediato, pero no agota las posibilidades de análisis en el nivel más impersonal de la dinámica de clases (Jameson, 1998, pp. 69-136); dicho de otro modo, estimo necesario tensionar la particularidad de las situaciones de clases específicas con la abstracción propia de la economía política.

En este sentido, remitiéndome a un ensayo de Eric Hobsbawm que leí por primera vez en aquel curso de 1993 (Hobsbawm, 2004, pp. 84-104), considero que, como ciencia de las sociedades humanas, la historia social -ahora ramificada en varias subdisciplinas especializadas - debe seguir asumiendo temas como los de clases y sociabilidades sin dejar de lado su perspectiva de conjunto -o, dicho en términos técnicos, de totalidad concreta (Kosik, 1976)-. Por ello mismo, insisto, el aporte del Dr. Acuña Ortega sigue siendo irrenunciable para la historia social actual en Centroamérica.

\section{BIBLIOGRAFÍA}

Acuña, Víctor Hugo (1993). "Clases subalternas y movimientos sociales en Centroamérica (18701930)", Historia general de Centroamérica, tomo IV. Madrid: FLACSO-Sociedad Estatal Quinto Centenario. 
Acuña, Víctor Hugo (1996). “La historia social en Costa Rica: balance y perspectivas”, Revista de historia (Costa Rica), número especial.

Acuña, Víctor Hugo (2007). Historia e incertidumbre. San José: EUCR, 2007.

Acuña, Víctor Hugo (2004). "The formation of the urban middle sectors in El Salvador, 19101944", en Aldo Lauria-Santiago y Leigh Binford (eds.). Landscapes of struggle. Politics, society and community in El Salvador. Pittsburgh: University of Pittsburgh Press.

Acuña, Víctor Hugo (1991). "Vida cotidiana, condiciones de trabajo y organización sindical: el caso de los zapateros en Costa Rica (1934-1955)", en Acuña Ortega, Víctor Hugo e Iván Molina. Historia económica y social de Costa Rica (1750-1950). San José: Porvenir.

Acuña Ortega, Víctor Hugo (1986). "Patrones del conflicto social en la economía cafetalera costarricense (1900-1948)", en Revista de ciencias sociales (Costa Rica) 31.

Acuña Ortega, Víctor Hugo (1994). "Nación y clase obrera en Centroamérica durante la época liberal (1870-1930)" en eds. Steven Palmer e Iván Molina, El paso del cometa. Estado, política social y culturas populares en Costa Rica (1800/1950). San José: Porvenir.

Acuña Ortega, Víctor Hugo (1993). "Clases subalternas y movimientos sociales en Centroamérica (1870-1930)" en Historia general de Centroamérica. Tomo 4. Las repúblicas agroexportadoras, ed. Víctor Hugo Acuña. Madrid: FLACSO-Sociedad Estatal Quinto Centenario.

Acuña Ortega, Víctor Hugo (1985). "Clases sociales y conflicto social en la economía cafetalera costarricense: productores contra beneficiadores, 1932-1936”, en Revista de Historia (Costa Rica) Especial.

Acuña Ortega, Víctor Hugo (1986). La Huelga bananera de 1934 (San José: CENAP-CEPAS, 1984). Del mismo autor: Los orígenes de la clase obrera en Costa Rica: las huelgas de 1920 por la jornada de ocho horas. San José: CENAP-CEPAS.

Arias Mora, Dennis (2011). Utopías de quietud. Cuestión autoritaria y violencia, entre las sombras del nazismo y del dilema antifascista (Costa Rica, 1933-1943). San José: EUNED.

Agulhon, Maurice (1994). Historia vagabunda. México: Instituto Mora.

Agulhon, Maurice (2009). El círculo burgués. Bs. Aires: Siglo XXI.

Grüner, Eduardo (2002). El fin de las pequeñas historias. De los estudios culturales al retorno (imposible) de lo trágico. Bs. Aires: Paidós.

Hobsbawm, Eric (2004). "De la historia social a la historia de la sociedad", en: Sobre la historia. Barcelona: Crítica.

Jameson, Fredric (1998). "Sobre los 'Estudios culturales"”, en Fredric Jameson y Slavoj Žižek, Estudios culturales. Reflexiones sobre el multiculturalismo. Bs. Aires: Paidós.

Karel Kosík (1976), Dialéctica de lo concreto. Estudios sobre los problemas del hombre y el mundo. México: Grijalbo. 
Marx, Karl (2003). El dieciocho brumario de Luis Bonaparte. Madrid: Alianza.

Molina, Iván (2005). "Cuestión social, literatura y dinámica electoral en Costa Rica”, en Ronny Viales Hurtado (ed), Pobreza e historia en Costa Rica, Pobreza e historia en Costa Rica. Determinantes estructurales y representaciones sociales del siglo XVII a 1950. San José: EUCR.

Molina, Iván (2007). Anticomunismo reformista. Competencia electoral y cuestión social en Costa Rica (1931-1948). San José: Ed. Costa Rica.

Noiriel, Gérard (1997). Sobre la crisis de la historia. Valencia: Cátedra.

Palmer, Steven (1999). “Adiós laissez-faire: la política social en Costa Rica (1880-1940)”, en Revista de historia de América, 124 (enero-junio).

Palmer, Steven (2002). “Confinamiento, mantenimiento del orden y surgimiento de la política social en Costa Rica, 1880-1935”, en Mesoamérica, n. 43 (junio).

Thompson, E.P. (1989). Formación de la clase obrera en Inglaterra, tomo I. Barcelona: Crítica.

Viales, Ronny J. (2005). "El régimen liberal de bienestar y la institucionalización de la pobreza en Costa Rica. 1870-1930". En: Viales, Ronny (Editor) Pobreza e historia en Costa Rica. Determinantes estructurales y representaciones sociales del siglo XVII a 1950. San José: EUCR, CIHAC, Posgrado Centroamericano en Historia, pp. 71-100.

\section{ACERCA DEL AUTOR}

George García. Licenciado en Filosofía y profesor en las Escuelas de Filosofía y de Estudios Generales de la Universidad de Costa Rica; egresado de la maestría académica en Historia de la Universidad de Costa Rica. 